\title{
Analysis on the Impact of the Blood Sugar Control Levels of Gestational Diabetes Mellitus on the Maternal and Infant Outcomes
}

\author{
Xueying Yang ${ }^{1, a}$, Qun $\mathrm{Li}^{1}{ }^{1,}$, Ruxia Liu ${ }^{1}$ \\ ${ }^{1}$ Department of Obstetrics and Gynecology, The Fourth People's Hospital of Jinan, Jinan, 250031, \\ China \\ ayangxueying@126.com \\ *Corresponding author liqun@163.com
}

Keywords: Pregnancy; Diabetes; Blood glucose control level; Outcomes

\begin{abstract}
Objective: To observe the impact of the blood sugar control levels of gestational diabetes mellitus on the maternal and infant outcomes. Methods: We select 78 cases of GDM pregnant women from August 2013 to August 2014 in our hospital, and they are divided into satisfied and not satisfied group according to the blood sugar control levels, and from the same period in hospital childbirth in normal pregnant women randomly we selected 60 patients as the control group, the GDM pregnant women and the clinical data of 60 cases of control group in pregnant women were analyzed. Results: The GDM pregnant women in general surgery cesarean delivery, preeclampsia, hydramnios, postpartum hemorrhage and the incidence of reproductive tract infections compared with control group differences were statistically significant $(\mathrm{P}<0.05)$; The satisfactory group of GDM pregnant women and the perinatal pregnancy complications son end are no statistically significant difference compared with the control group ( $\mathrm{P}>0.05)$; The GDM pregnant women are not satisfied with group preeclampsia, hydramnios, postpartum hemorrhage and genital tract infection and neonatal premature birth, macrosomia, hypoglycemia, and the incidence of high blood bilirubin compared with GDM pregnant women satisfied group differences were statistically significant $(\mathrm{P}<0.05)$. Conclusion: The GDM has serious threat to maternal and child health, its influence degree is closely related to blood glucose control level. They should be timely screening for diabetes during pregnancy, early diagnosis, active treatment, reduce the maternal complications and improving the prognosis of maternal and infant.
\end{abstract}

\section{Introduction}

In patients with gestational diabetes is refers to the sugar metabolic potential before pregnancy or normal glucose tolerance and in the process of pregnancy or sugar metabolic abnormalities was discovered for the first time. Gestational diabetes occurs in the second half of pregnancy commonly, easy cause obstetric complications, impact on maternal and infant is bigger, so the effective control of blood sugar is particularly important. In order to investigate the correlation between the gestational diabetes control blood sugar levels and outcomes, the author analyzed retrospectively the gestational diabetes, the relationship between blood glucose control and pregnancy outcomes, results will now report as follows.

\section{Materials and Methods}

\section{Materials}

We select from August 2013 to August 2014 in the hospital was diagnosed with gestational diabetes 78 pregnant women as the research object, both for mothers. According to blood glucose control is divided the patients into glucose control are not satisfied with satisfaction and blood glucose control group 39 cases, two groups of patients with basic data comparison difference has no statistical significance $(\mathrm{P}>0.05)$. Alternate sugar screening test is normal, without diabetes risk factors of 60 cases of healthy pregnant women as control group, the basic data there was no statistically significant difference compared with the former two groups $(\mathrm{P}>0.05)$, comparable. 


\section{The inclusion criteria}

The early pregnancy testing pregnant women fasting glucose are twice higher than 5.8 tendencies, L can diagnosis of GDM. On fasting blood sugar is normal, in the 24 to 28 weeks of pregnancy go GCT, $50 \mathrm{~g} \mathrm{p} 7.8 \mathrm{mmol} / \mathrm{L}$ for GCT is positive, positive for glucose tolerance test (OGTT), 75 g OGTT two anomalies diagnosed with GDM. After diagnosis are given simple diet control, diet + insulin treatment and moderate exercise, keep fasting glucose $5.6 \mathrm{mmol} / \mathrm{L}$ (below $100 \mathrm{mg} / \mathrm{dl}$ ), $2 \mathrm{~h}$ blood glucose after meal is in $6.7 \mathrm{mmol} / \mathrm{L}$ (below $120 \mathrm{mg} / \mathrm{dl}$ ), pregnant women without apparent hunger is satisfied, if the fasting and postprandial blood glucose $2 \mathrm{~h}$ at least one of the above standard to control.

\section{Statistical processing}

With mathematical statistics software SPSS19.0 for data collection and statistical analysis, and chi-square test, $\mathrm{P}<0.05$, significant difference has statistical significance.

\section{Results}

The GDM pregnant women in general surgery cesarean delivery, preeclampsia, hydramnios, postpartum hemorrhage and the incidence of reproductive tract infections compared with control group differences were statistically significant $(\mathrm{P}<0.05)$; The satisfactory group of GDM pregnant women and the perinatal pregnancy complications son end are no statistically significant difference compared with the control group $(\mathrm{P}>0.05)$; The GDM pregnant women are not satisfied with group preeclampsia, hydramnios, postpartum hemorrhage and genital tract infection and neonatal premature birth, macrosomia, hypoglycemia, and the incidence of high blood bilirubin compared with GDM pregnant women satisfied group differences were statistically significant $(\mathrm{P}<0.05)$. (See Table 1 and Table 2)

Table 1: The influence of the GDM pregnant women blood sugar control levels on pregnancy outcome

\begin{tabular}{|c|c|c|c|c|c|c|c|}
\hline & Group & $\mathrm{n}$ & $\begin{array}{c}\text { Before the } \\
\text { operation }\end{array}$ & Preeclampsia & Hydramnios & $\begin{array}{l}\text { Postpartum } \\
\text { hemorrhage }\end{array}$ & $\begin{array}{c}\text { Genital } \\
\text { infection }\end{array}$ \\
\hline \multirow{3}{*}{$\begin{array}{c}\text { The GDM } \\
\text { group }\end{array}$} & $\begin{array}{l}\text { The satisfactory } \\
\text { group }\end{array}$ & 39 & 22 & 2 & 3 & 4 & 2 \\
\hline & $\begin{array}{l}\text { The unsatisfactory } \\
\text { group }\end{array}$ & 39 & 32 & 10 & 14 & 13 & 11 \\
\hline & ntrol group & 60 & 29 & 1 & 2 & 3 & 3 \\
\hline
\end{tabular}

Table 2: The influence of the GDM pregnant women blood sugar control level on the perinatal outcome

\begin{tabular}{|c|c|c|c|c|c|c|c|}
\hline & Group & $\mathrm{n}$ & $\begin{array}{l}\text { Preterm } \\
\text { birth }\end{array}$ & Macrosomia & Hypoglycemia & $\begin{array}{l}\text { Neonatal } \\
\text { asphyxia }\end{array}$ & $\begin{array}{l}\text { High bilirubin } \\
\text { blood disease }\end{array}$ \\
\hline \multirow{2}{*}{$\begin{array}{l}\text { The } \\
\text { GDM } \\
\text { group }\end{array}$} & $\begin{array}{c}\text { The } \\
\text { satisfactory } \\
\text { group }\end{array}$ & 39 & 1 & 2 & 4 & 1 & 3 \\
\hline & $\begin{array}{l}\text { The } \\
\text { unsatisfactory } \\
\text { group }\end{array}$ & 39 & 6 & 12 & 19 & 5 & 16 \\
\hline \multicolumn{2}{|c|}{ Control group } & 60 & 1 & 4 & 3 & 0 & 4 \\
\hline
\end{tabular}

\section{Discussion}

\section{The clinical manifestations of gestational diabetes}

Number of patients with gestational diabetes hair more and more in recent years in our country, their sugar metabolism in postpartum can return to normal, but increase the chance of future type 2 diabetes. Involved in glucose metabolism during pregnancy has many liters of blood sugar hormone, along with the process of pregnancy, these rises gradually increased blood sugar hormone 
production, imbalance between the hormone insulin and insulin resistance. Various hormone insulin resistance in $24 \sim 28$ weeks pregnancy when rapidly rising, peaked in $32 \sim 34$ weeks gestation, routine screening for diabetes during pregnancy as $24 \sim 28$ weeks of pregnancy, this stage of the exception can diagnosis of gestational diabetes. Gestational diabetes cause pregnant women to metabolic disorder, not only lead to many diseases in pregnancy, and fetal development is adverse, could lead to premature birth, neonatal hypoglycemia, neonatal asphyxia, etc. Research has shown that gestational diabetes control blood sugar levels can affect pregnancy outcomes. If blood glucose control during pregnancy, will seriously affect and outcomes. Gestational diabetes pregnant state of sustained high blood sugar can cause small vascular endothelial cell proliferation, extensive vascular lesions, lumen narrowing, tissue blood supply is reduced, occurrence of gestational hypertension significantly increased. Mother's high insulin hematic disease will increase fetal oxygen consumption, inhibition of lung surface active substance, the synthesis of lung mature delay, and intrauterine distress and neonatal asphyxia. Fetal hyperglycemia will cause high permeability diuresis, hydramnios, premature rupture of membranes. At present, there are two main types of pregnant women blood sugar control methods: diet therapy and drug therapy (mainly insulin treatment). The research thinks, insulin effect is better than oral medications, improve the effect of the pregnancy outcome of gestational diabetes, blood sugar control of the ideal gestational hypertension, polyhydramnios, premature rupture of membranes, postpartum hemorrhage, neonatal asphyxia, the incidence of premature infant and neonatal hypoglycemia was obviously lower than blood glucose control is not ideal., therefore, should be timely for gestational diabetes screening and diagnosis, and through the reasonable diet and insulin therapy, the blood sugar control in a reasonable level, it is very important for maternal and infant security.

\section{The gestational diabetes affect pregnant women and perinatal}

The GDM patients because of poor blood sugar control, combined vascular pathological changes make it easier for pregnant women complicated with gestational hypertension disease. Due to the pregnant women with gestational diabetes blood glucose, high sugar content in the amniotic fluid, stimulate the secretion of amniotic membrane increased, because hydramnios, prone to premature rupture of membranes, lead to premature birth, premature birth and low birth weight. Zhang Yujie thinks $2 \mathrm{~h}$ after meals such as blood sugar levels and birth weight were positively correlated relationship. Postprandial hyperglycemia through the placenta into the fetal body, can cause fetal blood sugar increased, stimulate fetal islet beta cell hyperplasia, hypertrophy and insulin secretion increased, to adjust the fetal blood sugar. Fetal high insulin hematic disease, promote the synthesis of tissue metabolism and growth and development, leading to the occurrence of macrosomia. Pregnant women of sustained high blood sugar can reduce the blood oxygen supply of placenta to the fetus; In addition, increase tissue oxygen consumption, fetal hyperinsulinemia lead to fetal hypoxia, also easily lead to premature birth. As with many research results, this group of data results support the above conclusion. This data shows poor blood sugar control, gestational hypertension, polyhydramnios, premature rupture of membranes, preterm, macrosomia, incidence of neonatal asphyxia increased significantly.

GDM patients blood sugar control are not satisfied, easily complicated with macrosomia, hydramnios, postpartum hemorrhage and therefore are more likely to occur. High blood sugar is good microbial culture medium, are prone to cause wound infection, wound to heal; Diabetes patients of microcirculation is poorer, capillary embolization, poor blood supply, the wound site did not have enough nutrients, therefore not easy to heal. But the GDM pregnant women after the termination of pregnancy, blood sugar can be back to normal. Due to the observation of the number of cases is less, in this information on the relationship between the blood glucose levels and postpartum wound infection remains to be determined. Because of good medical environment and the use of antibiotics to prevent postpartum infection effectively, in the observation cases occurred postpartum wound infections found in 2 cases.

Fetal intrauterine distress for many reasons, this study found that the blood sugar level is to mark, but at the same time and other complications, so that the high incidence of fetal distress. Fetal 
distress is one of the important reasons, amniotic fluid dung dye dyeing rate is two groups of pregnant women in the amniotic fluid dung to standard set of high blood glucose control, but there was no statistically significant difference. ICP for late in pregnancy, unique complications occurred, now think that the onset of ICP may be associated with high estrogen during pregnancy. Estrogen can stimulate the liver to produce cortisol binding globulin (joined). When joined quantity increase, pregnant women more cortisol adrenal glands to complement more joined the required. At the same time increase blood free cortisol causes high cortisol, high cortisol levels can be against insulin and glucose clearance delay, indirectly play a role of insulin resistance, the ICP easily complicated with GDM pregnant women. Subjective symptoms by ICP, is often a pregnant women see a doctor, so combining ICP earlier treatment, timely find the GDM and easier to control blood sugar.

Gestational diabetes is not associated absolute surgical indications, but the GDM pregnant women complicated with gestational hypertension disease, diabetic ketoacidosis, fetal distress, macrosomia complications, such as the opportunity increase. But this study shows that two groups of cesarean delivery rate of GDM patients with blood sugar control target group is more, but there is no statistical difference. This is because in the social factors for the surgical indications.

The GDM is on the rise in recent years, mother-baby complication is more, there are mainly natural abortion, pregnancy hypertension disease, premature birth, infection, fetal intrauterine growth restriction, macrosomia, fetal distress, and low blood sugar, etc., and even serious impact on maternal and infant. For GDM has made great progress in the study of, blood sugar control satisfaction of maternal and perinatal mortality basic with GDM pregnant women approached, but among disease rate and around the sick rate is still high, therefore, need to strengthen the management of GDM pregnancy, reduce maternal and infant complications.

\section{The early diagnosis and early control hyperglycemia to reduce the complications and improve the prognosis of the most important factors}

Good control of blood glucose levels in pregnant women who gestational diabetes can effectively reduce the occurrence of complications, is very important to the safety of the maternal and infant. Gestational diabetes, pregnant women are the main serious complications of genital tract infection and postpartum hemorrhage, the high incidence of $20 \%$. Postpartum hemorrhage is one of the pregnant women four major causes of death, should be paying attention. For the influence of the newborn is high blood bilirubin. High blood bilirubin is a multifactor disease, survivors two-thirds have permanent neurological sequelae, and obvious gestational diabetes is a risk factor for high blood bilirubin. Hydramnios incidence rate is about $0.5 \% \sim 1 \%$, the incidence of patients with diabetes mellitus is as high as $20 \%$, and no obvious symptoms. Hydramnios of cesarean delivery, placental abruption, maternal postpartum hemorrhage, fetal abnormalities, death, umbilical cord prolapsed, and fetal umbilical cord around the neck and larger than normal maternal amniotic fluid. This study showed that blood glucose control is not satisfied with GDM pregnant women in newborns, macrosomia, premature neonatal hypoglycemia and incidence of high blood bilirubin was obviously higher than that of blood sugar control group and normal pregnant women, consistent with literature reports. Research confirmed that the rate of perinatal disease blood glucose levels was positively correlated with the pregnant woman. Pregnant women can reduce high blood sugar itself the placenta to the fetus blood oxygen supply, lead to fetal hypoxia, especially pregnant women with gestational hypertension disease, is adding to the fetal hypoxia, acidosis, and an increased incidence of fetal distress and neonatal asphyxia. Can cause fetal hyperglycemia and hyperinsulinemia fetal lung II cell surface active material to reduce, increase the occurrence of neonatal respiratory distress syndrome; After birth, if not timely supplement increased incidence of low blood sugar. Fetal metabolism caused by increasing stimulates fetal bone marrow hematopoietic erythrocytes, lead to high blood bilirubin.

The gestational diabetes pathogenesis inducement is not entirely clear, but studies have shown that the GDM have familial transmissibility, may be associated with genetic and other nutritional factors of obesity is the major cause, increased BMI before pregnancy can predict the GDM pregnant women need insulin therapy. Because the etiology is not clear, only can use treats the 
method of controlling the blood sugar levels, such as diet and exercise therapy, insulin injections when necessary, in general, not recommended for oral insulin.

\section{Conclusion}

This study found that the earlier diagnosis and treatment time actively cooperate with treatment, are more likely to the maintain blood glucose control in the range of satisfaction, the gestational hypertension, premature rupture of membranes, hydramnios, postpartum hemorrhage, premature infants, neonatal asphyxia, the incidence of macrosomia under poor control of blood sugar. Therefore strengthen the pregnant women health care, early detection and diagnosis of diabetes mellitus, timely and effectively control blood sugar, is critical, is to reduce the complications and improve the prognosis of the most important factors.

\section{Acknowledgement}

This research was financially supported by the National Science Foundation.

\section{References}

[1] Tal Sella, Varda Shalev, Uriel Elchalal, Aluma Chovel-Sella, Gabriel Chodick. Screening for gestational diabetes in the 21 st century: a population-based cohort study in Israel[J]. Journal of Maternal-Fetal and Neonatal Medicine . 2013 (4)

[2] Michella Ghassibe-Sabbagh ,Daniel E. Platt,Sonia Youhanna, Antoine B. Abchee, Krista Stewart,Danielle A. Badro, Marc Haber, Angelique K. Salloum, Bouchra Douaihy, Hamid el Bayeh, Raed Othman, Nabil Shasha, Samer Kibbani, Elie Chammas, Aline Milane, Rita Nemr, Yoichiro Kamatani, Jörg Hager, Jean-Baptiste Cazier, Dominique Gauguier, Pierre A. Zalloua. Genetic and environmental influences on total plasma homocysteine and its role in coronary artery disease risk[J]. Atherosclerosis . 2012 (1)

[3] Vikas Veeranna, Sandip K. Zalawadiya,Ashutosh Niraj,Jyotiranjan Pradhan,Brian Ference,Robert C. Burack,Sony Jacob,Luis Afonso. Homocysteine and Reclassification of Cardiovascular Disease Risk[J]. Journal of the American College of Cardiology. 2011 (10)

[4] Natalia Úbeda,Leticia Reyes, Antonio González-Medina,Elena Alonso-Aperte,Gregorio Varela-Moreiras. Physiologic changes in homocysteine metabolism in pregnancy: A longitudinal study in Spain[J]. Nutrition . 2011 (9)

[5] Camila Simioni Vanzin, Giovana Brondani Biancini, Angela Sitta, Carlos Alberto Yasin Wayhs, Izabela Netto Pereira, Francieli Rockenbach, Solange Cristina Garcia, Angela Terezinha de Souza Wyse,Ida Vanessa Doederlein Schwartz,Moacir Wajner,Carmen Regla Vargas. Experimental evidence of oxidative stress in plasma of homocystinuric patients: A possible role for homocysteine[J]. Molecular Genetics and Metabolism . 2011 (1)

[6] B. Idzior-Waluś, K. Cyganek, K. Sztefko, G. Seghieri, M. C. Breschi,M. Waluś-Miarka, E. Kawalec, M. Seretny, J. Sieradzki. Total plasma homocysteine correlates in women with gestational diabetes[J]. Archives of Gynecology and Obstetrics . 2008 (4)

[7] Ingrid P.Krapels, Christl Vermeij - Keers, Michael Müller, Annelies Klein, Régine P.Steegers-Theunissen. Nutrition and Genes in the Development of Orofacial Clefting $[\mathrm{J}]$. Nutrition Reviews . 2008 (6)

[8] Vinukonda Govindaiah, Shaik Mohammad Naushad, Krishnamurthy Prabhakara, Prasad Chintakindi Krishna,Akella Radha Rama Devi. Association of parental hyperhomocysteinemia and C677T Methylene tetrahydrofolate reductase (MTHFR) polymorphism with recurrent pregnancy loss[J]. Clinical Biochemistry . 2008 (4)

[9] Yumi Masuda, Akira Kubo, Akatsuki Kokaze, Masao Yoshida, Nobuki Fukuhara,Yutaka Takashima. Factors associated with serum total homocysteine level in type 2 diabetes[J]. Environmental Health and Preventive Medicine . 2008 (3) 
[10] M. A. Guven,M. Kilinc, C. Batukan,H. C. Ekerbicer, T. Aksu. Elevated second trimester serum homocysteine levels in women with gestational diabetes mellitus[J]. Archives of Gynecology and Obstetrics . 2006 (6) 